\title{
Effect of screening on spectroscopic properties of Li-like ions in plasma environment
}

\author{
Pradip Kumar Mondal, ${ }^{1}$ Narendra Nath Dutta, ${ }^{1}$ Gopal Dixit, ${ }^{2, *}$ and Sonjoy Majumder ${ }^{1,+}$ \\ ${ }^{1}$ Department of Physics and Meteorology Indian Institute \\ of Technology Kharagpur, Kharagpur-721302, India \\ ${ }^{2}$ Center for Free-Electron Laser Science, DESY, \\ Notkestrasse 85, 22607 Hamburg, Germany
}

(Dated: June 5, 2013)

\begin{abstract}
This work presents accurate $a b$ initio investigations of various spectroscopic properties of a few Li-like ions in presence of a plasma environment within the Debye screening potential. The coupledcluster theory in the relativistic framework has been employed to compute ionization potentials, excitation energies, electric dipole oscillator strengths, and electric quadrupole transition probabilities of Li-like $\mathrm{C}^{3+}, \mathrm{N}^{4+}$, and $\mathrm{O}^{5+}$ ions. The unretarded Breit interaction has been implemented to increase the accuracy of the calculations. The effects of ion density and temperature on the ionization potentials, excitation energies, electric dipole oscillator strengths, and electric quadrupole transition probabilities have been investigated in the plasma environment. It is found that the plasma screening leads to a sharp decrease in the ionization potential as the screening strength increases. With increasing strength, the oscillator strengths associated with $2 s^{2} S_{1 / 2} \rightarrow 2 p^{2} P_{1 / 2,3 / 2}$ transitions increase, whereas the transition probabilities associated with $3 d^{2} D_{3 / 2,5 / 2} \rightarrow 2 s{ }^{2} S_{1 / 2}$ transitions decrease.
\end{abstract}

PACS numbers:

* gopal.dixit@cfel.de

tsonjoy@gmail.com 


\section{INTRODUCTION}

With the advent of novel x-ray sources based on free-electron lasers (FELs) [1, 2], laser plasmas [3], and high-harmonic generations [4, 5], it is possible to achieve extreme conditions in matter such as high energy density and high temperature using ultraintense, ultrashort, and tunable pulses, and hence it is possible to create matter in plasma form [6, 7]. There have been many experimental [6-16] and theoretical [17-35] endeavors to explain and understand the effect of plasma environment on the spectroscopic properties of atoms and ions. In the situation, when atoms or ions embedded in plasma, the interaction between the nucleus and the bound electrons is screened by the surrounding ions and fast electrons. The modified interaction gives rise to phenomena such as pressure ionization and continuum lowering and affects the spectroscopic properties of atoms and ions [36, 37]. Recent advanced experiment, carried out using FEL and electron beam ion trap (EBIT), provides an unexpected low oscillator strength of electric dipole $(E 1)$ transition of $\mathrm{Fe}^{16+}$ and raises the concern about the quality of the atomic wave functions used to model such spectral properties [38]. Therefore, treating the effect of plasma environment in atoms and ions along with an accurate treatment of electron-electron correlation and relativistic effects are nontrivial. The ratio of Coulomb energy to thermal energy determine the strength of coupling $(\Gamma)$ in plasma. The low density and high temperature situation corresponds to weakly coupled plasma $(\Gamma<1)$, where the screening of the nuclear Coulomb interaction by free electrons in the plasma is guided by the Debye model [39, 40].

Lithium and lithium-like ions in plasma are a few of the most abundant ionic species for specific temperature and density attainable in the laboratory [41]. Various spectroscopic properties of Li-like ions have significant importance in astrophysics due to evidence of high abundances of these ions in different astronomical systems like active galactic nuclei, x-ray binaries, quasars, and hot plasmas [42, 43]. For such small-sized atoms, allowed and forbidden transitions with sufficient intensity are used as diagnostic tools of tokamak plasmas [44-46]. It is well known that the ionized form of carbon, nitrogen, and oxygen and their various transition lines are important for the chromosphere region of the solar atmosphere [47], and to understand the dynamics and nature of the stellar and interstellar medium [48 52]. There is long literature on the applications of the isolated resonance lines having wavelength $1548.19 \AA$ and $1550.77 \AA$ for $\mathrm{C}^{3+}, 1238.82 \AA$ and $1242.80 \AA$ for 
$\mathrm{N}^{4+}$ and $1031.91 \AA$ and $1037.61 \AA$ for $\mathrm{O}^{5+}$ [53, 54]. The astronomical observed lines are expected to be affected by the plasma atmosphere at the origin and therefore important for plasma diagnostic purpose. Forbidden transition lines, i.e., electric quadrupole $(E 2)$ and magnetic dipole $(M 1)$ transition lines provide very crucial parameters for estimations of density and internal temperature measurements at low density hot plasmas [55 57]. Also, the transition rates of forbidden transitions provide accurate dielectric recombination rates for these ions [58 60].

Several theoretical methods have been used to model the effect of plasma environment on the spectroscopic properties for one-electron [22, 24, 29] and many-electron [27, 28, 61 63] systems. Due to the screening effect, lowering of the ionization potential is demonstrated by Stewart and Pyatt [64]. The Debye plasma screening on lighter atoms or ions have been studied over last decade using different many-body approaches [27-29, 65] and showed enough avenues of improvement. Recent works of correlation exhaustive Dirac-Coulomb based coupled -cluster calculations on He-, Li-, Be- and Na-like ions [61 63] are examples of this. The authors of these works emphasized the importance of relativistic correction on the plasma screening by using more accurate many-body theories within a relativistic framework $[61-63]$.

In the present article, we analyze the influence of the plasma screening on the Li-like $\mathrm{C}^{3+}$, $\mathrm{N}^{4+}$, and $\mathrm{O}^{5+}$ ions using the Debye model potential. The ionization potentials, excitation energies, oscillator strengths of $E 1$ transitions, and transition rates of $E 2$ transitions are estimated for these ions in the isolated (free) condition as well as within the plasma environment. Here, we have used the Fock-space coupled-cluster (FSCC) method within the Dirac-Coulomb-Breit (DCB) Hamiltonian to consider the relativistic effect on these spectral properties. Recently, Dutta et al. have implemented the unretarded Breit interaction in an all-order approach using the coupled-cluster (CC) theory and demonstrate the effect of electron-electron correlation and unretarded Breit interaction on the boron isoelectronic sequence [66]. It is well known that the dynamical electron correlation, relaxation effect, and Breit interaction are important for moderately charged ions, which are considered here in an accurate way. This paper is structured as follows. Section II discusses brief theory and formalism of the relativistic FSCC approach with the Debye screening potential. Section III presents results and discussions on several spectral properties of Li-like ions and the effect of plasma screening on these properties. Conclusions and future outlooks are presented in 
Sec. IV.

\section{THEORY}

In order to consider the effect of plasma environment on the spectroscopic properties, the Dirac-Coulomb (DC) Hamiltonian with unretarded Breit interaction for an $N$-electron atomic system can be written as

$$
H=\sum_{i=1}^{N}\left(c \overrightarrow{\alpha_{i}} \cdot \overrightarrow{p_{i}}+\left(\beta_{i}-1\right) c^{2}+V_{\mathrm{eff}}^{D}\left(r_{i}\right)+\sum_{j<i}\left(\frac{1}{r_{i j}}-\frac{\overrightarrow{\alpha_{i}} \cdot \overrightarrow{\alpha_{j}}}{r_{i j}}\right)\right),
$$

with all the standard notations often used. Here, $V_{\text {eff }}^{D}\left(r_{i}\right)$ is the effective potential of the nucleus on the $i$-th electron due to the presence of plasma environment. The Debye-Hückel potential is considered to examine the effect of screening of nuclear Coulomb potential due to the presence of ions and free electrons in plasmas [39, 67]. In case of weakly interacting plasma medium, the effective potential experienced by the $i$-th electron is given as

$$
V_{\text {eff }}^{D}\left(r_{i}\right)=-\frac{Z e^{-\mu r_{i}}}{r_{i}},
$$

where $Z$ is the nuclear charge and $\mu$ is the Debye screening parameter, which is related to the ion density $n_{\text {ion }}$ and plasma temperature $T$ through the following relation:

$$
\mu=\left[\frac{4 \pi(1+Z) n_{\mathrm{ion}}}{k_{B} T}\right]^{\frac{1}{2}},
$$

where $k_{B}$ is the Boltzmann constant. Therefore, a given value of $\mu$ represents a range of plasma conditions with different ion densities and temperatures. The inverse of the Debye screening parameter is called the Debye screening length, i.e., $\lambda_{D}=\mu^{-1}$. The pure Coulomb nuclear attraction corresponds to the zero screening situation $(\mu=0)$.

The wave functions, ionization potentials (IPs) of the ground, and the different excited states for the considered $N$-electron atomic system are obtained using the FSCC method with single, double, and partial triple excitations within the relativistic framework. The basic formalism of the FSCC method was developed several decades before [68-72]. The relativistic version of the FSCC theory has been developed recently and successfully employed to obtain the various properties in different single valence atomic systems [33 80]. Here, we provide key steps of this method. In the relativistic coupled-cluster (RCC) theory, the correlated 
wave function of a single valence atomic state with a valance electron in the " $v$ " th orbital is written in the form,

$$
\left|\Psi_{v}\right\rangle=e^{T}\left\{1+S_{v}\right\}\left|\Phi_{v}\right\rangle
$$

where, $\left|\Phi_{v}\right\rangle$ is the corresponding reference state generated at the Dirac-Fock (DF) level of the $N-1$ electron closed-shell system using Koopman's theorem [81]. T represents all possible excitations from the core orbitals of the closed-shell system, and $S_{v}$ represents all possible valence and core-valence excitations of the single-valence system. The detail inclusion of the unretarded Breit interaction in this formalism is described by Dutta and Majumder [66].

The transition matrix element for any operator $O$ in the framework of the RCC wave function can be expressed as

$$
\begin{aligned}
O_{f i} & =\frac{\left\langle\Psi_{f}|O| \Psi_{i}\right\rangle}{\sqrt{\left\langle\Psi_{f} \mid \Psi_{f}\right\rangle\left\langle\Psi_{i} \mid \Psi_{i}\right\rangle}} \\
& =\frac{\left\langle\Phi_{f}\left|\left\{1+S_{f}^{\dagger}\right\} e^{T^{\dagger}} O e^{T}\left\{1+S_{i}\right\}\right| \Phi_{i}\right\rangle}{\sqrt{\left\langle\Phi_{f}\left|\left\{1+S_{f}^{\dagger}\right\} e^{T^{\dagger}} e^{T}\left\{1+S_{f}\right\}\right| \Phi_{f}\right\rangle\left\langle\Phi_{i}\left|\left\{1+S_{i}^{\dagger}\right\} e^{T^{\dagger}} e^{T}\left\{1+S_{i}\right\}\right| \Phi_{i}\right\rangle}} .
\end{aligned}
$$

The single-electron reduced matrix elements corresponding to $E 1, E 2$, and $M 1$ transitions are discussed in Refs. [82, 83].

The transition probabilities (in $\mathrm{s}^{-1}$ ) corresponding to $E 1, E 2$, and $M 1$ channels from state $k$ to $i$ is given as

$$
\begin{aligned}
& A_{k \rightarrow i}^{E 1}=\frac{2.0261 \times 10^{18}}{\lambda^{3} g_{k}} S^{E 1}, \\
& A_{k \rightarrow i}^{E 2}=\frac{1.11995 \times 10^{18}}{\lambda^{5} g_{k}} S^{E 2}
\end{aligned}
$$

and

$$
A_{k \rightarrow i}^{M 1}=\frac{2.69735 \times 10^{13}}{\lambda^{3} g_{k}} S^{M 1},
$$

where $S=\left|\left\langle\Psi_{k}|O| \Psi_{i}\right\rangle\right|^{2}$ is the transition strength of the operator $O$ (in a.u.), $\lambda$ (in $\AA$ ) is the corresponding transition wavelength, and $g_{k}=2 j_{k}+1$ is the degeneracy of the $k$ state. The oscillator strength of $E 1$ transition from state $i$ to $k$ is given as

$$
f_{i \rightarrow k}^{E 1}=1.4992 \times 10^{-16} \frac{g_{k}}{g_{i}} \lambda^{2} A_{k \rightarrow i}^{E 1} .
$$

\section{RESULTS AND DISCUSSIONS}

The precise DF orbital wave functions, which are the building block of accurate correlation calculations, are generated via the basis set expansion technique in the potential of two core 
electrons at $1 s_{1 / 2}$ orbital. The radial part of these basis wave functions are considered to be Gaussian type [84] having two optimized exponential parameters $\alpha_{0}$ and $\beta$. The nuclei are considered as finite size with a Fermi-type charge distribution [85]. For all the ions considered here, the number of basis wave functions at the DF levels for $s^{-}, p-, d-$, and $f$-type symmetries are 30, 25, 20, and 20, respectively. In order to choose the optimized parameters for each ion, the energies and wave functions of the DF orbitals are compared with the same as obtained from the GRASP92 code, where the DF equations are solved numerically [86]. These exponential parameters are chosen as 0.005825 and 2.73 for $\mathrm{C}^{3+}$, 0.003265 and 2.73 for $\mathrm{N}^{4+}$, and 0.00525 and 2.73 for $\mathrm{O}^{5+}$. The number of $\mathrm{DF}$ orbitals for different symmetries used in the RCC calculations are based on the convergent criteria of core correlation energies with increasing number of orbitals. There are 12, 11, 10, and 10 active orbitals, which include all core orbitals, considered in the calculations for the $s, p$, $d$, and $f$ type symmetries, respectively. As an improvement of the atomic Hamiltonian beyond the Dirac-Coulomb limit, the Breit interaction in it's unretarded approximation has been included for more accurate relativistic descriptions of the wave functions [66]. The $T$ amplitudes are solved first for the closed-shell systems and later the $S$ amplitudes corresponding to different single-valence states are solved for the open-shell systems using the RCC equations.

The quality of the wave functions for different eigen states is ensured by comparing the reduced matrix elements of the $E 1$ transitions in length and velocity gauges [83]. The calculated average deviations of these matrix elements between these two gauges at the CC levels have been found $1.11 \%$ for $\mathrm{C}^{3+}, 1.17 \%$ for $\mathrm{N}^{4+}$, and $1.58 \%$ for $\mathrm{O}^{5+}$, which indicate very good quality of the relativistic wave functions. The calculated values of the ground state IPs for $\mathrm{C}^{3+}, \mathrm{N}^{4+}$, and $\mathrm{O}^{5+}$ are 520145, 789494 and $1113968 \mathrm{~cm}^{-1}$, respectively; which are in excellent agreement with the NIST results: 520178, 789537 and $1114004 \mathrm{~cm}^{-1}$, respectively. The average deviations of our calculated excitation energies (EEs) of different excited states with respect to the NIST results are estimated around $0.05 \%, 0.06 \%$ and $0.06 \%$ for $\mathrm{C}^{3+}$, $\mathrm{N}^{4+}$, and $\mathrm{O}^{5+}$, respectively [54].

The E1 oscillator strengths are presented in Table \along with the available NIST results [54] for comparison. The average deviations between our calculated results and the NIST values are about $1.6 \%$ for $\mathrm{C}^{3+}, 1.2 \%$ for $\mathrm{N}^{4+}$, and $1.2 \%$ for $\mathrm{O}^{5+}$. Our results for oscillator strength agree excellently with the recent FSCC results presented by Das et al. for 
$\mathrm{C}^{3+}$ ion [62]. The recently calculated results of Elabidi et al. using SUPERSTRUCTURE code [87] have an average deviation of about $3 \%$ with respect to the NIST results; though, in some cases deviations are around 25\% [87]. Therefore, correlation exhaustive and relativistic calculations were wanted, and our calculations are motivated towards that. The oscillator strengths of $3 d^{2} D_{3 / 2,5 / 2} \rightarrow 4 f^{2} F_{5 / 2,7 / 2}$ transitions are estimated here as well.

Table II presents the emission probabilities of $E 2$ transitions having values of the order of $10^{4} \mathrm{~s}^{-1}$ or more using precisely calculated wavelengths for $\mathrm{C}^{3+}, \mathrm{N}^{4+}$, and $\mathrm{O}^{5+}$. Sur and Chaudhuri have reported a few E2 transition probabilities for $\mathrm{O}^{5+}$ using the RCC theory based on the DC Hamiltonian [88]. Their results differ by about $0.8 \%$ from our calculated values obtained by the same theory but based on the DCB Hamiltonian. All the transitions presented in the Table II fall in the ultraviolet region of electromagnetic spectrum. The wavelengths of these forbidden lines relative to those of allowed lines from the same ion make them very good candidates for line profile measurements and help to understand the excitation processes, like electron- and proton-impact excitations [89, 90]. It is evident from the table that there are few strong E2 transitions with transition probabilities of the order of $10^{6} \mathrm{~s}^{-1}$. These are the transitions between the ground states and $3 d^{2} D_{3 / 2,5 / 2}$ states for $\mathrm{N}^{4+}$, and $\mathrm{O}^{5+}$, and the transitions $3 p^{2} P_{1 / 2} \rightarrow 2 p^{2} P_{3 / 2}$ and $4 f^{2} F_{5 / 2} \rightarrow 2 p^{2} P_{1 / 2}$ for $\mathrm{O}^{5+}$. Therefore, these forbidden transition lines having relatively higher probabilities can play in the determinations of density and internal temperature inside hot plasmas. Because the $M 1$ transition probabilities are found to be quite low (of the order of $10^{-1} \mathrm{~s}^{-1}$ or less), the lines associated with these transitions are hardly possible to detect and hence, are excluded from the consideration here.

After validating the quality and accuracy of our method and calculations for unscreened ions, i.e., with $\mu=0$, several spectroscopic properties for Li-like ions are calculated for different values of Debye screening parameter $\mu$. In most of the scenario, the ions are presented in low density plasmas where all the spectroscopic properties are affected by the plasma atmosphere which are quantified by the Debye screening parameter $\mu$. We have chosen the values of $\mu$ ranging from 0 to 0.175 a.u. in interval of 0.025 a.u.. The ions become unstable after $\mu=0.175$ a.u.. It is mentioned already in the "Theory" ection that $\mu$ is a function of ion density $n_{\text {ion }}$ and plasma temperature $T$. Therefore, the above ranges of $\mu$ mimic weakly coupled plasma; for example, $T=10^{6} \mathrm{~K}$ and $n_{\text {ion }} \sim 10^{22} \mathrm{~cm}^{-3}$ correspond to $\mu=0.15$ a.u. [62, 91]. This type of condition can be achieved in the laboratory plasmas 
TABLE I: Oscillator strengths of $E 1$ transitions and their comparisons with the NIST results for $\mathrm{C}^{3+}, \mathrm{N}^{4+}$, and $\mathrm{O}^{5+}$.

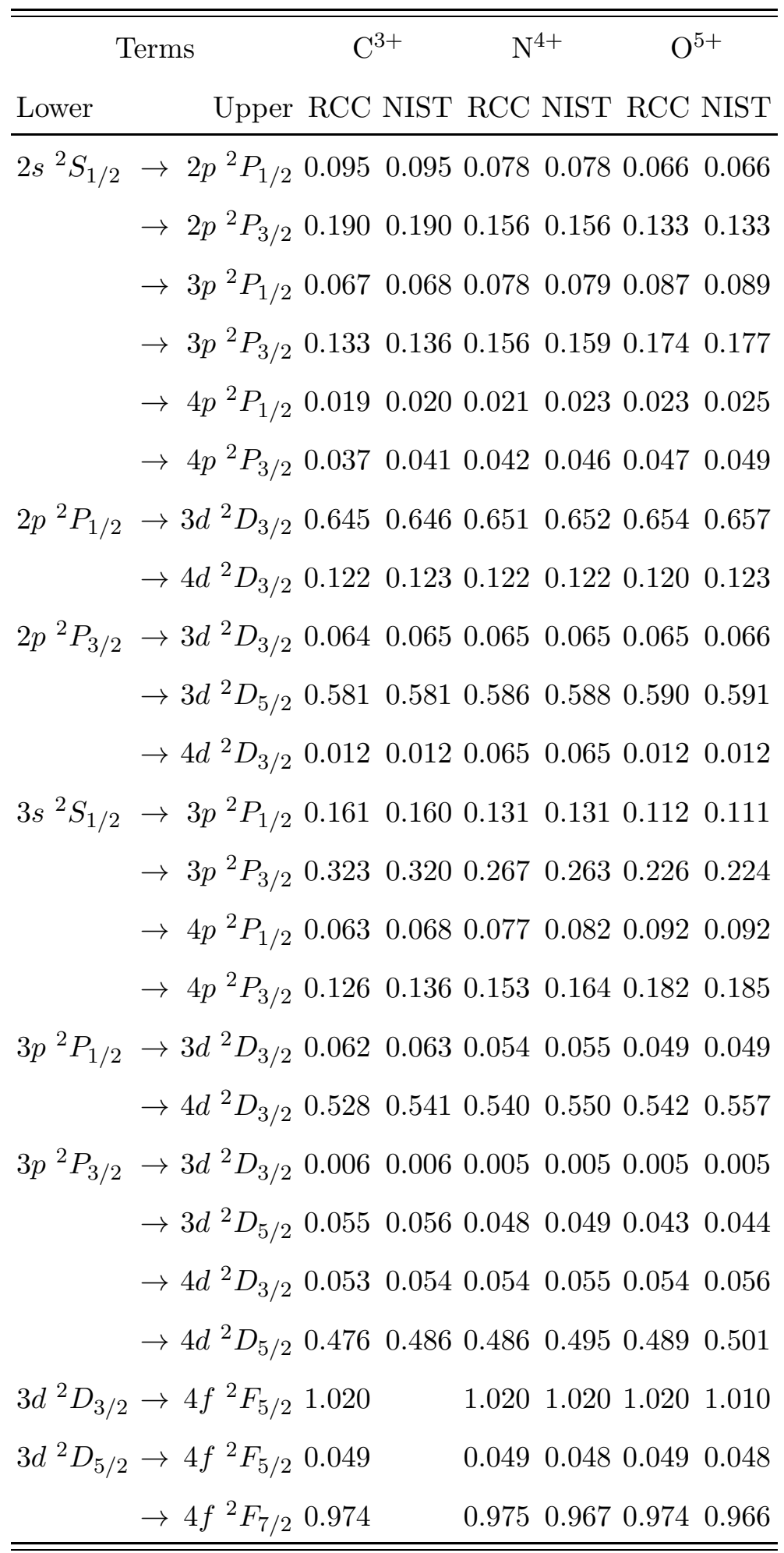

for high temperature [91]. The relative variations in the IPs as a function of $\mu$ for $\mathrm{C}^{3+}, \mathrm{N}^{4+}$, 
TABLE II: Transition probabilities (in $10^{4} \mathrm{~s}^{-1}$ ) of $E 2$ transitions along with corresponding transition wavelengths (in $\AA$ ) for $\mathrm{C}^{3+}, \mathrm{N}^{4+}$, and $\mathrm{O}^{5+}$.

\begin{tabular}{|c|c|c|c|c|c|c|c|c|c|}
\hline \multirow[b]{3}{*}{ Upper } & \multicolumn{3}{|c|}{$\mathrm{C}^{3+}$} & \multicolumn{3}{|c|}{$\mathrm{N}^{4+}$} & \multicolumn{3}{|c|}{$\mathrm{O}^{5+}$} \\
\hline & \multicolumn{2}{|c|}{$\lambda$} & \multirow[t]{2}{*}{$A_{i f}$} & \multicolumn{2}{|r|}{$\lambda$} & \multirow[t]{2}{*}{$A_{i f}$} & \multicolumn{2}{|c|}{$\lambda$} & \multirow[t]{2}{*}{$A_{i f}$} \\
\hline & $\mathrm{RCC}$ & NIST & & $\mathrm{RCC}$ & NIST & & $\mathrm{RCC}$ & NIST & \\
\hline $3 d^{2} D_{3 / 2} \rightarrow 2 s^{2} S_{1 / 2}$ & 307.71 & 307.81 & 44.03 & 206.36 & & 150.17 & 148.16 & & 413.17 \\
\hline $3 d^{2} D_{5 / 2} \rightarrow 2 s^{2} S_{1 / 2}$ & 307.70 & 307.79 & 44.04 & 206.35 & 206.43 & 150.23 & 148.15 & 148.21 & 413.41 \\
\hline $4 d^{2} D_{3 / 2} \rightarrow 2 s^{2} S_{1 / 2}$ & 243.39 & 243.71 & 10.33 & 161.62 & 161.83 & 30.06 & 115.21 & 115.35 & 78.05 \\
\hline $4 d^{2} D_{5 / 2} \rightarrow 2 s^{2} S_{1 / 2}$ & 243.39 & 243.71 & 12.30 & 161.61 & 161.83 & 30.09 & 115.21 & 115.35 & 78.14 \\
\hline $3 p^{2} P_{3 / 2} \rightarrow 2 p^{2} P_{1 / 2}$ & 391.08 & 391.23 & 5.31 & 251.52 & 251.64 & 20.01 & 175.42 & 175.47 & 59.53 \\
\hline $4 p^{2} P_{3 / 2} \rightarrow 2 p^{2} P_{1 / 2}$ & 290.56 & 290.83 & 1.91 & 186.81 & 187.01 & 7.19 & 130.32 & 130.37 & 22.87 \\
\hline $4 f^{2} F_{5 / 2} \rightarrow 2 p^{2} P_{1 / 2}$ & 289.03 & & 19.99 & 185.99 & 186.01 & 76.16 & 129.72 & & 228.22 \\
\hline $3 p^{2} P_{1 / 2} \rightarrow 2 p^{2} P_{3 / 2}$ & 391.32 & 391.45 & 10.61 & 251.76 & 251.86 & 40.01 & 175.65 & 175.68 & 118.97 \\
\hline $3 p^{2} P_{3 / 2} \rightarrow 2 p^{2} P_{3 / 2}$ & 391.26 & 391.40 & 5.30 & 251.71 & 251.81 & 19.99 & 175.61 & 175.63 & 59.46 \\
\hline $4 p^{2} P_{1 / 2} \rightarrow 2 p^{2} P_{3 / 2}$ & 290.68 & 290.93 & 3.82 & 186.92 & 187.11 & 14.35 & 130.43 & 130.47 & 45.62 \\
\hline $4 p^{2} P_{3 / 2} \rightarrow 2 p^{2} P_{3 / 2}$ & 290.66 & 290.92 & 1.91 & 186.91 & 187.11 & 7.18 & 130.42 & 130.46 & 22.83 \\
\hline $4 f^{2} F_{5 / 2} \rightarrow 2 p^{2} P_{3 / 2}$ & 289.13 & & 5.71 & 186.09 & & 21.76 & 129.82 & 129.84 & 65.20 \\
\hline $4 d^{2} D_{3 / 2} \rightarrow 3 s^{2} S_{1 / 2}$ & 926.31 & 930.34 & 2.36 & 615.27 & 618.08 & 8.57 & 438.96 & 440.83 & 24.74 \\
\hline $4 d^{2} D_{5 / 2} \rightarrow 3 s^{2} S_{1 / 2}$ & 926.27 & 930.30 & 2.36 & 615.23 & 618.05 & 8.57 & 438.92 & 440.79 & 24.74 \\
\hline $4 p^{2} P_{3 / 2} \rightarrow 3 p^{2} P_{1 / 2}$ & 1130.07 & 1132.83 & 0.54 & 725.71 & 727.75 & 2.02 & 506.47 & 506.88 & 6.18 \\
\hline $4 f^{2} F_{5 / 2} \rightarrow 3 p^{2} P_{1 / 2}$ & 1107.17 & 1106.38 & 2.22 & 713.41 & 712.79 & 8.29 & 497.54 & 497.61 & 24.40 \\
\hline $4 p^{2} P_{1 / 2} \rightarrow 3 p{ }^{2} P_{3 / 2} 1$ & 1130.75 & 1133.41 & 1.07 & 726.35 & & 4.04 & 507.10 & 507.44 & 12.36 \\
\hline $4 p^{2} P_{3 / 2} \rightarrow 3 p{ }^{2} P_{3 / 2} 1$ & 1130.54 & 1133.24 & 0.54 & 726.16 & 728.16 & 2.02 & 506.91 & 507.28 & 6.18 \\
\hline $4 f^{2} F_{5 / 2} \rightarrow 3 p^{2} P_{3 / 2} 1$ & 1107.62 & 1106.77 & 0.63 & 713.84 & 713.86 & 2.36 & 497.96 & 497.98 & 6.95 \\
\hline $4 f^{2} F_{7 / 2} \rightarrow 3 p^{2} P_{3 / 2} 1$ & 1107.61 & 1106.77 & 2.85 & 713.82 & 713.15 & 10.64 & 497.94 & 497.96 & 31.29 \\
\hline $4 d^{2} D_{3 / 2} \rightarrow 3 d^{2} D_{3 / 2} 1$ & 1164.01 & 1170.18 & 0.33 & 745.01 & 748.99 & 1.27 & 517.81 & 520.14 & 3.80 \\
\hline $4 d^{2} D_{5 / 2} \rightarrow 3 d^{2} D_{3 / 2} 1$ & 1163.95 & 1170.13 & 0.095 & 744.95 & 748.94 & 0.36 & 517.75 & 520.08 & 1.08 \\
\hline $4 d^{2} D_{3 / 2} \rightarrow 3 d^{2} D_{5 / 2} 1$ & 1164.13 & 1170.33 & 0.14 & 745.13 & 749.11 & 0.55 & 517.93 & 520.28 & 1.63 \\
\hline $4 d^{2} D_{5 / 2} \rightarrow 3 d^{2} D_{5 / 2} 1$ & 1164.08 & 1170.27 & 0.38 & 745.08 & 749.06 & 1.41 & 517.88 & 520.22 & 4.34 \\
\hline
\end{tabular}


and $\mathrm{O}^{5+}$ ions are presented in Fig. 1. For convenience, we define relative variation in any spectroscopic property of interest here, say $\mathbf{O}$, as

$$
\text { Relative variation in } \mathbf{O}=\frac{[\mathbf{O}(\mu \neq 0)-\mathbf{O}(\mu=0)] \times 100}{\mathbf{O}(\mu=0)} .
$$

It is evident from the Fig. 1 that as the ion density increases or temperature decreases, i.e., the screening strength $\mu$ increases, IPs decrease linearly and the systems become less and less stable. This is because, the screening of the nuclear charge increases with the increase of $\mu$ and hence, the attractive nuclear Coulomb potential at the valence electron decreases. This particular fact can be attributed as continuum lowering for the system surrounded in plasma environment. A similar trend was observed already for Li and Li-like ions in the presence of Debye plasma environment [62, 91]. In addition, this figure shows that the fall in IP decreases with increasing ionic charge, i.e., the IP of $\mathrm{C}^{3+}$ decreases more rapidly than the IPs of $\mathrm{N}^{4+}$, and $\mathrm{O}^{5+}$ within a same interval of $\mu$. With increasing nuclear charge $Z$ of an isoelectronic sequence, the valence electron comes closer to the nucleus and hence can defend more effectively the screening of the plasma environment.

Figure 2 presents the relative variations in the excitation energies (EEs) of different lowlying excited states for $\mu=0.075$ a.u. for the Li-like ions. For this value of $\mu$, the effect of screening on the EEs is found optimum. However, we have observed similar trends in the EEs for any other values of $\mu$ within the given range of weakly coupled plasma. It is evident from this figure that due to the screening, the ground-state transitions from the states $2 p^{2} P_{1 / 2,3 / 2}$ are blue-shifted, and from the others are red-shifted. This figure further reflects that the shift in the EEs decreases as the nuclear charge increases. These shifts in the EEs can be attributed by the quantum confinement and electron screening in the presence of the plasma environment [28]. It is well known that the quantum defect decreases as the orbital angular momentum quantum number increases, and for angular momentum quantum number equal and larger than two, the quantum defect is almost zero. Therefore, electronic states with higher angular momentum quantum number among the same principle quantum number experience relatively less effect of $\mu$. For example, EEs for $3 d^{2} D_{3 / 2,5 / 2}$ are less perturbed than the states $3 s^{2} S_{1 / 2}$ and $3 p^{2} P_{1 / 2,3 / 2}$ due to screening. In addition, the effect of quantum confinement is the same for the fine structure states. The figure further confirms that the quantum confinement is more pronounced for higher excited states than relatively low-lying states [28]. 


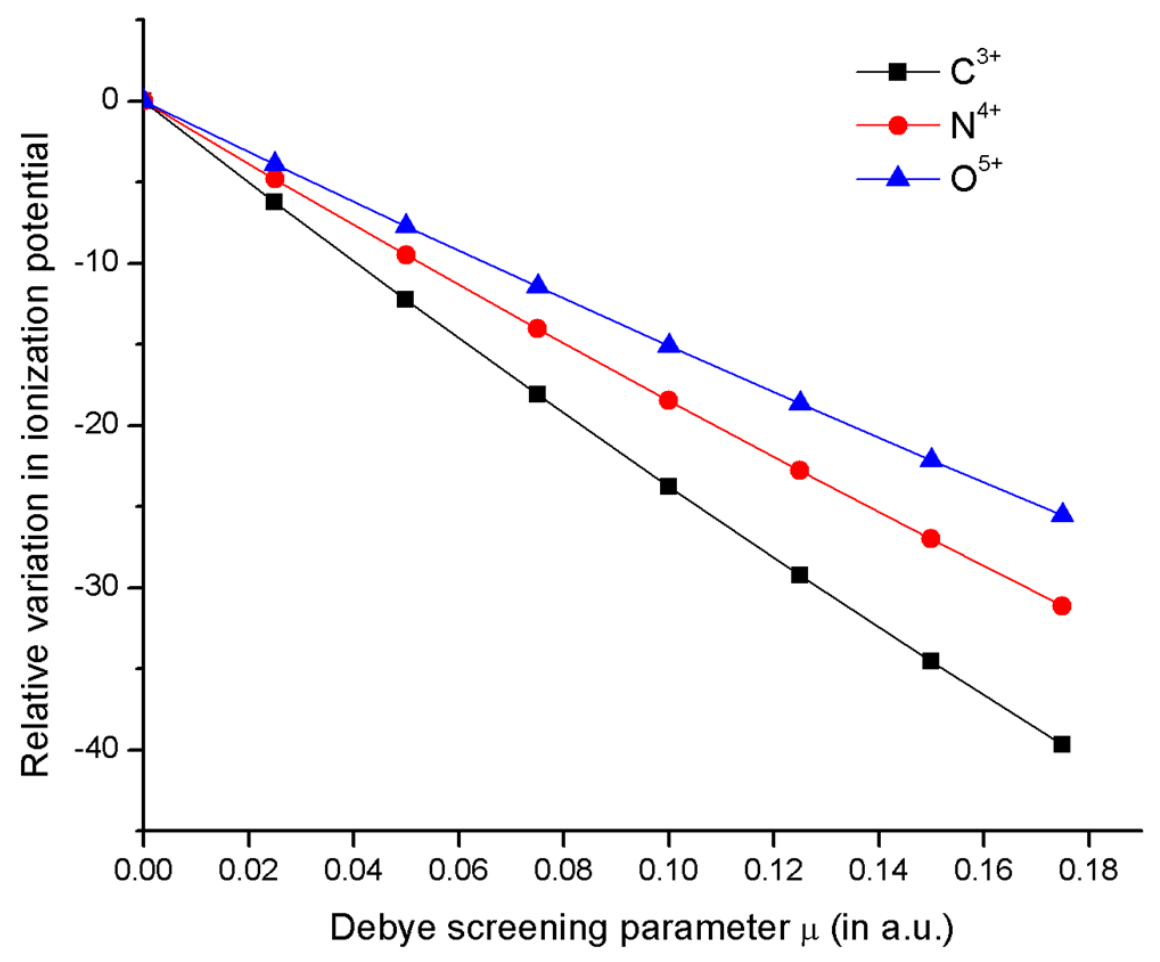

FIG. 1: (Color online) Relative variations of ionization potentials with Debye screening parameter $\mu$ for $\mathrm{C}^{3+}, \mathrm{N}^{4+}$, and $\mathrm{O}^{5+}$.

The relative variations of oscillator strengths with respect to $\mu$ values for the most strong $2 s{ }^{2} S_{1 / 2} \rightarrow 2 p{ }^{2} P_{1 / 2,3 / 2}$ transitions of $\mathrm{C}^{3+}, \mathrm{N}^{4+}$, and $\mathrm{O}^{5+}$ ions are shown in Fig. 22 The relative variations increase monotonically as $\mu$ increases and the effect is the same for both the transitions. For a particular value of $\mu$, the screening effect on the oscillator strengths decreases as nuclear charge increases. However, the transition energies corresponding to these transitions change significantly, which reflect in the total change in the oscillator strengths as observed in Fig. 3. This present trend of the $E 1$ oscillator strengths in Debye plasma has been reported in the recent past [35, 62]. Our present findings show that the lifetimes of $2 \mathrm{p}^{2} P_{1 / 2,3 / 2}$ states decrease with increasing plasma strength $\mu$. The lifetimes of these states depend on the third power of wavelengths of the associated $E 1$ transitions to the ground state. This enhances the screening effect on the lifetimes with respect to the oscillator strengths which depend inversely on the first power of the corresponding wavelengths.

The transition probabilities $\left(A^{E 2}\right)$ of the $E 2$ transition $3 d^{2} D_{3 / 2} \rightarrow 2 s^{2} S_{1 / 2}$ along with the corresponding transition wavelengths for different values of $\mu$ are presented in Table III. It 


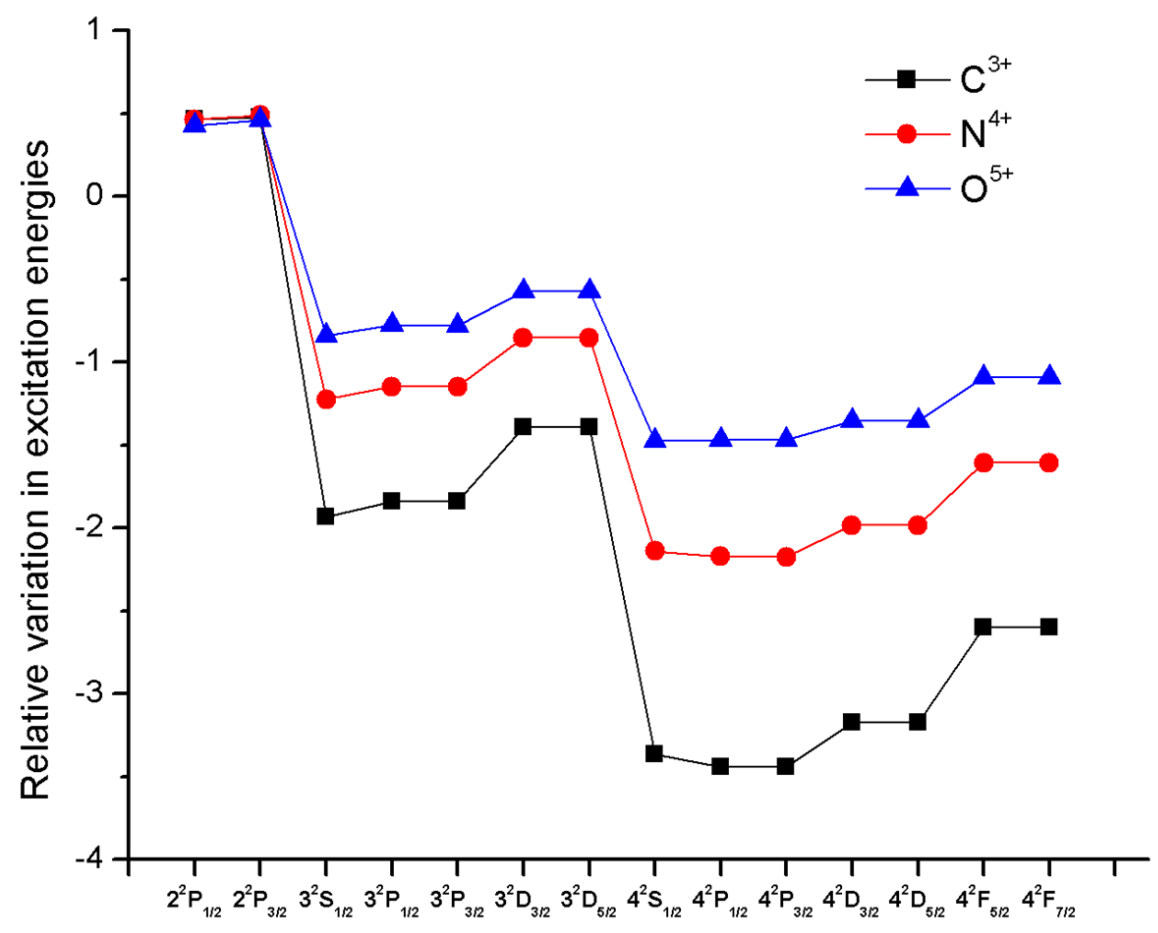

\section{Electronic states}

FIG. 2: (Color online) Relative variations of excitation energies of different excited states with Debye screening parameter $\mu=0.075$ a.u. for $\mathrm{C}^{3+}, \mathrm{N}^{4+}$, and $\mathrm{O}^{5+}$.
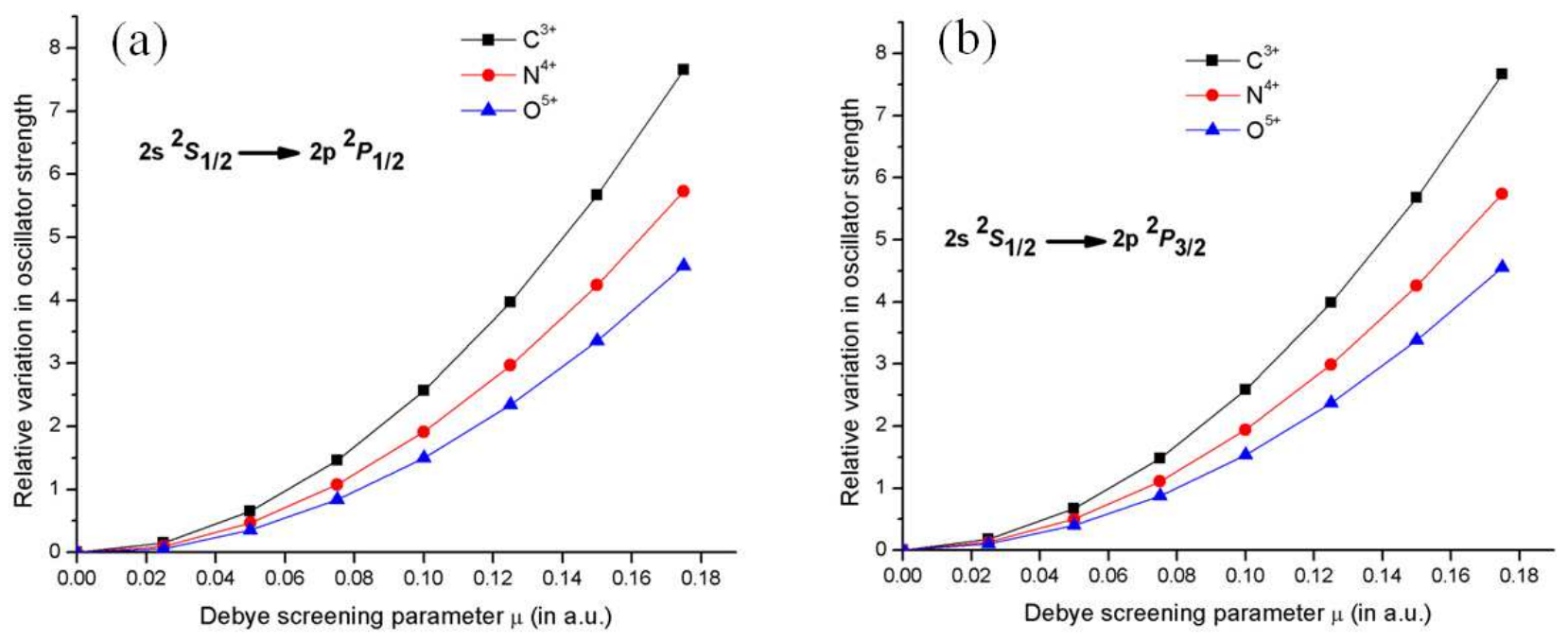

FIG. 3: (Color online) Relative variations of oscillator strengths of $E 1$ transitions with Debye screening parameter $\mu$ for $\mathrm{C}^{3+}, \mathrm{N}^{4+}$, and $\mathrm{O}^{5+}$. Panels (a) and (b) correspond to $2 s^{2} S_{1 / 2} \rightarrow 2 p^{2} P_{1 / 2}$ and $2 s^{2} S_{1 / 2} \rightarrow 2 p{ }^{2} P_{3 / 2}$ transitions, respectively. 
is evident from the table that the $E 2$ transition wavelengths are red shifted with increasing value of the screening strength. A similar trend is observed for the $3 d^{2} D_{5 / 2} \rightarrow 2 s{ }^{2} S_{1 / 2}$ transition, which is not presented here. The relative variations in the transition probabilities for both these $E 2$ transitions as a function of $\mu$ are depicted in Fig. 4. Both these transitions are chosen due to their comparatively higher probabilities with respect to other transitions as evident from Table II. The systematic decrease of the transition probabilities with increasing $\mu$ is observed from the figure. One can also visualize that the relative decrease in $E 2$ transition probabilities for a particular value of screening parameter is more for $\mathrm{C}^{3+}$ compared to $\mathrm{O}^{5+}$. If one compares the relative changes in $E 1$ versus $E 2$ transition amplitudes as a function of $\mu$, one finds that the latter is more influenced by $\mu$ than the former. This behavior can be explained from their radial dependence. The amplitude of $E 1$ transition has $r$ dependence, where that of $E 2$ transition has $r^{2}$ dependence. Due to this, E2 transition amplitude depends on further field region compared to $E 1$ transition amplitude from the nucleus and hence, E2 transition amplitude is relatively more affected by the screening. At this point, it is important to emphasis that $E 2$ transitions are the effective processes in the low density hot plasmas. Therefore, estimations of the influence of nuclear charge screening in the E2 transition probabilities along with their wavelengths are of great importance in plasma modeling.

TABLE III: Effect of $\mu$ (in a.u.) on $A_{3 d^{2} D_{3 / 2} \rightarrow 2 s^{2} S_{1 / 2}}$ (in $10^{4} \mathrm{~s}^{-1}$ ) and corresponding transition wavelengths (in $\AA$ ) for $\mathrm{C}^{3+}, \mathrm{N}^{4+}$, and $\mathrm{O}^{5+}$.

\begin{tabular}{|c|c|c|c|c|c|}
\hline \multirow{2}{*}{$\mu$} & $\mathrm{C}^{3+}$ & \multicolumn{2}{|c|}{$\mathrm{N}^{4+}$} & \\
\hline & $\lambda \quad A^{E 2}$ & $\lambda$ & $\lambda \quad A^{E 2}$ & & $A^{E 2}$ \\
\hline 0 & 307.7144 .03 & 206.36 & 6150.17 & 148.16 & 413.17 \\
\hline 0.025 & 308.2243 .68 & 206.57 & 7149.47 & 148.26 & 411.92 \\
\hline 0.05 & 309.6942 .70 & 207.18 & 8147.46 & 148.56 & 408.29 \\
\hline 0.075 & 312.1041 .14 & 208.17 & 7144.26 & 149.04 & 402.46 \\
\hline 0.1 & 315.4439 .06 & 209.53 & 3139.95 & 149.70 & 394.56 \\
\hline 0.125 & 319.7536 .48 & 211.27 & 7134.62 & 150.54 & 384.72 \\
\hline 0.15 & 325.1333 .42 & 213.40 & O 128.27 & 151.56 & 373.04 \\
\hline 0.175 & 331.7129 .87 & 215.94 & 4121.00 & 152.76 & 359.60 \\
\hline
\end{tabular}



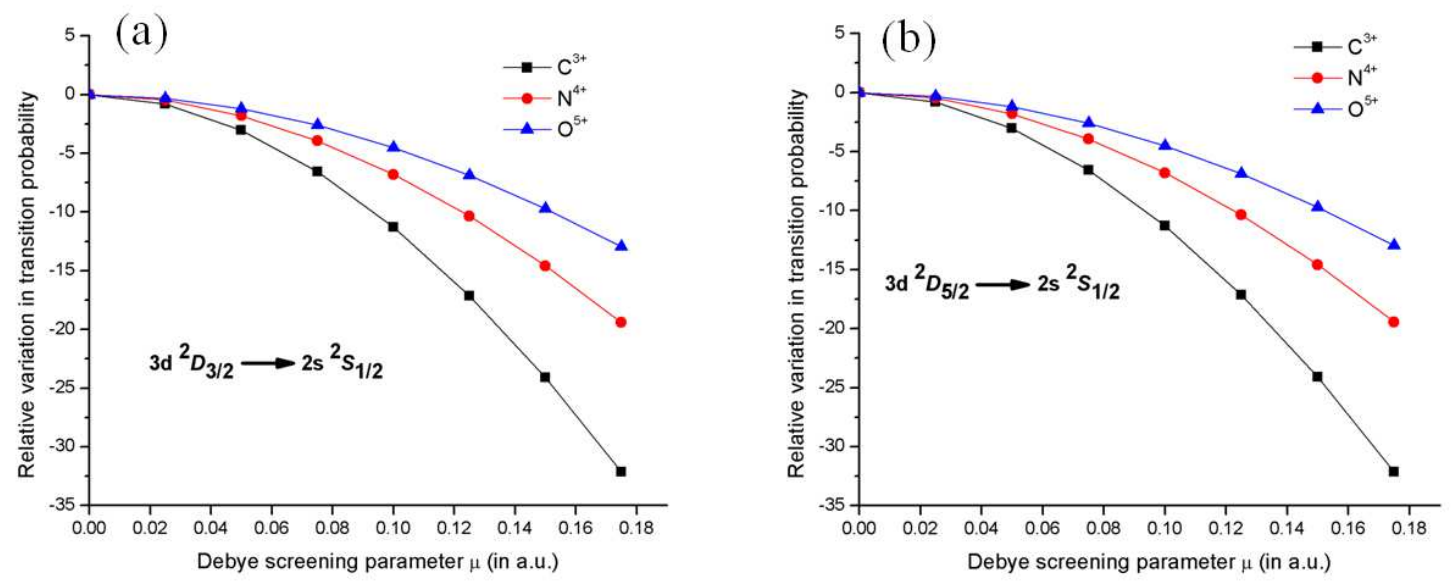

FIG. 4: (Color online) Relative variations of transition probabilities of $E 2$ transitions with Debye screening parameter $\mu$ for $\mathrm{C}^{3+}, \mathrm{N}^{4+}$, and $\mathrm{O}^{5+}$. Panels (a) and (b) correspond to $3 d^{2} D_{3 / 2} \rightarrow$ $2 s^{2} S_{1 / 2}$, and (b) $3 d^{2} D_{5 / 2} \rightarrow 2 s{ }^{2} S_{1 / 2}$ transitions, respectively.

\section{CONCLUSION}

We have investigated the influence of Debye screening of nuclear charges due to the presence of free electrons and ions in plasma medium on the ionization potentials, excitation energies, E1 oscillator strengths, and E2 transition probabilities of $\mathrm{C}^{3+}, \mathrm{N}^{4+}$, and $\mathrm{O}^{5+}$. Especially, the study on E2 transitions can be considered a useful tool to model low density and high temperature plasmas. The transition wavelengths are mainly affected by this screening, which characterizes the screening effects on the other associated spectroscopic properties like the oscillator strengths and transition probabilities. Due to the high abundances of these Li-like ions in various astrophysical systems, we hope our investigations will be useful to the astrophysicist in the near future. 


\section{Acknowledgments}

Pradip Kumar Mondal and Narendra Nath Dutta recognize financial help from the Council of Scientific and Industrial Research (CSIR), India.

[1] P. Emma, R. Akre, J. Arthur, R. Bionta, C. Bostedt, J. Bozek, A. Brachmann, P. Bucksbaum, R. Coffee, F. J. Decker, Y. Ding, D. Dowell, S. Edstrom, A. Fisher, J. Frisch, S. Gilevich, J. Hastings, G. Hays, P. Hering, Z. Huang, R. Iverson, H. Loos, M. Messerschmidt, A. Miahnahri, S. Moeller, H. D. Nuhn, G. Pile, D. Ratner, J. Rzepiela, D. Schultz, T. Smith, P. Stefan, H. Tompkins, J. Turner, J. Welch, W. White, J. Wu, G. Yocky, and J. Galayda, Nature Photonics 4, 641 (2010).

[2] T. Ishikawa, H. Aoyagi, T. Asaka, Y. Asano, N. Azumi, T. Bizen, H. Ego, K. Fukami, T. Fukui, Y. Furukawa, S. Goto, H. Hanaki, T. Hara, T. Hasegawa, T. Hatsui, A. Higashiya, T. Hirono, N. Hosoda, M. Ishii, T. Inagaki, Y. Inubushi, T. Itoga, Y. Joti, M. Kago, T. Kameshima, H. Kimura, Y. Kirihara, A. Kiyomichi, T. Kobayashi, C. Kondo, T. Kudo, H. Maesaka, X. M. Marechal, S. Masuda, T.and Matsubara, T. Matsumoto, T. Matsushita, S. Matsui, M. Nagasono, N. Nariyama, H. Ohashi, T. Ohata, T. Ohshima, S. Ono, Y. Otake, C. Saji, T. Sakurai, T. Sato, K. Sawada, T. Seike, K. Shirasawa, T. Sugimoto, S. Suzuki, S. Takahashi, H. Takebe, K. Takeshita, K. Tamasaku, H. Tanaka, R. Tanaka, T. Tanaka, T. Togashi, K. Togawa, A. Tokuhisa, H. Tomizawa, K. Tono, S. K. Wu, M. Yabashi, M. Yamaga, A. Yamashita, K. Yanagida, C. Zhang, T. Shintake, H. Kitamura, and N. Kumagai, Nature Photonics 6, 540 (2012).

[3] A. Rousse, C. Rischel, and J. C. Gauthier, Rev. Mod. Phys. 73, 17 (2001).

[4] I. McKinnie and H. Kapteyn, Nature Photonics 4, 149 (2010).

[5] T. Popmintchev, M. C. Chen, D. Popmintchev, P. Arpin, S. Brown, S. Ališauskas, G. Andriukaitis, T. Balčiunas, O. D. Mücke, A. Pugzlys, A. Baltuska, B. Shim, S. E. Schrauth, A. Gaeta, C. Hernandez-Garcia, L. Plaja, A. Becker, A. Jaron-Becker, M. M. Murnane, and H. C. Kapteyn, Science 336, 1287 (2012).

[6] S. M. Vinko, O. Ciricosta, B. I. Cho, K. Engelhorn, H. K. Chung, C. R. D. Brown, T. Burian, J. Chalupskỳ, R. W. Falcone, C. Graves, V. Hajkova, A. Higginbotham, L. Juha, J. Krzywin- 
ski, H. J. Lee, M. Messerschmidt, Y. Murphy, C. D. Ping, A. Scherz, W. Schlotter, S. Toleikis, J. J. Turner, L. Vysin, T. Wang, B. Wu, U. Zastrau, D. Zhu, R. W. Lee, P. A. Heimann, B. Nagler, and J. S. Wark, Nature 482, 59 (2012).

[7] O. Ciricosta, S. M. Vinko, H. K. Chung, B. I. Cho, C. R. D. Brown, T. Burian, J. Chalupskỳ, K. Engelhorn, R. W. Falcone, C. Graves, V. Hajkova, A. Higginbotham, L. Juha, J. Krzywinski, H. J. Lee, M. Messerschmidt, C. D. Murphy, Y. Ping, D. S. Rackstraw, A. Scherz, W. Schlotter, S. Toleikis, J. J. Turner, L. Vysin, T. Wang, B. Wu, U. Zastrau, D. Zhu, R. W. Lee, P. Heimann, B. Nagler, and J. S. Wark, Phys. Rev. Lett. 109, 65002 (2012).

[8] M. Murnane, H. Kapteyn, M. Rosen, and R. Falcone, Science 251, 531 (1991).

[9] D. Riley, L. A. Gizzi, F. Y. Khattak, A. J. Mackinnon, S. M. Viana, and O. Willi, Physical Review Letters 69, 3739 (1992).

[10] G. Mourou and D. Umstadter, Physics of Fluids B 4, 2315 (1992).

[11] F. J. Rogers and C. A. Iglesias, Science 263, 50 (1994).

[12] K. Nazir, S. J. Rose, A. Djaoui, G. J. Tallents, M. G. Holden, P. A. Norreys, P. Fews, J. Zhang, and F. Failles, Applied Physics Letters 69, 3686 (1996).

[13] J. Workman, M. Nantel, A. Maksimchuk, and D. Umstadter, Applied Physics Letters 70, 312 (1997).

[14] M. Nantel, G. Ma, S. Gu, C. Y. Cote, J. Itatani, and D. Umstadter, Physical Review Letters 80, 4442 (1998).

[15] N. C. Woolsey, B. A. Hammel, C. J. Keane, C. A. Back, J. C. Moreno, J. K. Nash, A. Calisti, C. Mosse, R. Stamm, B. Talin, A. Asfaw, L. S. Klein, and R. W. Lee, Physical Review E 57, 4650 (1998).

[16] A. Saemann, K. Eidmann, I. E. Golovkin, R. C. Mancini, E. Andersson, E. Förster, and K. Witte, Physical Review Letters 82, 4843 (1999).

[17] C. A. Rouse, Physical Review A 4, 90 (1971).

[18] U. Gupta and A. K. Rajagopal, Physics Reports 87, 259 (1982).

[19] J. Seidel, S. Arndt, and W. Kraeft, Physical Review E 52, 5387 (1995).

[20] D. Ray and P. K. Mukherjee, Journal of Physics B 31, 3479 (1998).

[21] Y. D. Jung, European Physical Journal D 7, 249 (1999).

[22] D. Ray, Physical Review E 62, 4126 (2000).

[23] J. Pang, G. Han, Z. Wu, and S. Li, Journal of Physics B 35, 2117 (2002). 
[24] B. Saha, P. K. Mukherjee, and G. H. F. Diercksen, Astronomy and Astrophysics 396, 337 (2002).

[25] H. Okutsu, T. Sako, K. Yamanouchi, and G. Diercksen, Journal of Physics B 38, 917 (2005).

[26] B. Saha, P. K. Mukherjee, D. Bielinska-Waz, and J. Karwowski, Journal of Quantitative Spectroscopy and Radiative Transfer 92, 1 (2005).

[27] B. Saha and S. Fritzsche, Physical Review E 73, 036405 (2006).

[28] Y. Li, J. Wu, Y. Hou, and J. Yuan, Journal of Physics B 41, 145002 (2008).

[29] S. Bhattacharyya, A. N. Sil, S. Fritzsche, and P. K. Mukherjee, European Physical Journal D 46, 1 (2008).

[30] J. K. Saha, S. Bhattacharyya, T. K. Mukherjee, and P. K. Mukherjee, Journal of Physics B 42, 245701 (2009).

[31] S. Paul and Y. K. Ho, Physics of Plasmas 16, 063302 (2009).

[32] Y. Y. Qi, Y. Wu, J. G. Wang, and Y. Z. Qu, Physics of Plasmas 16, 023502 (2009).

[33] A. N. Sil, J. Anton, S. Fritzsche, P. K. Mukherjee, and B. Fricke, European Physical Journal D 55, 645 (2009).

[34] C. Gao, J. Zeng, and J. Yuan, High Energy Density Physics 7, 54 (2011).

[35] L. Y. Xie, J. G. Wang, R. K. Janev, Y. Z. Qu, and C. Z. Dong, European Physical Journal D 66, 125 (2012).

[36] H. R. Griem, Spectral Line Broadening by Plasmas, New York, Academic Press, 1974.

[37] W. Jaskólski, Physics Reports 271, 1 (1996).

[38] S. Bernitt, G. V. Brown, J. K. Rudolph, R. Steinbrgge, A. Graf, M. Leutenegger, S. W. Epp, S. Eberle, K. Kubicek, V. Mckel, M. C. Simon, E. Trbert, E. W. Magee, C. Beilmann, N. Hell, S. Schippers, A. Mller, S. M. Kahn, A. Surzhykov, Z. Harman, C. H. Keitel, J. Clementson, F. S. Porter, W. Schlotter, J. J. Turner, J. Ullrich, P. Beiersdorfer, and J. R. Crespo LpezUrrutia, Nature 492, 225 (2012).

[39] S. Ichimaru, Reviews of Modern Physics 54, 1017 (1982).

[40] M. S. Murillo and J. C. Weisheit, Physics Reports 302, 1 (1998).

[41] T. Kawachi, T. Fujimoto, and G. Csanak, Physical Review E 51, 1428 (1995).

[42] S. N. Nahar, A. K. Pradhan, and H. L. Zhang, The Astrophysical Journal Supplement Series 131, 375 (2000).

[43] T. W. Gorczyca, I. Dumitriu, M. F. Hasoğlu, K. T. Korista, N. R. Badnell, D. W. Savin, and 
S. T. Manson, The Astrophysical Journal Letters 638, L121 (2006).

[44] S. Suckewer, H. P. Eubank, R. J. Goldston, J. McEnerney, N. R. Sauthoff, and H. H. Towner, Nuclear Fusion 21, 1301 (1981).

[45] M. Godefroid and C. F. Fischer, Journal of Physics B 17, 681 (1984).

[46] G. C. Das and J. Sarma, Physics of Plasmas 5, 3918 (1998).

[47] J. M. Fontenla, K. S. Balasubramaniam, and J. Harder, The Astrophysical Journal 667, 1243 (2007).

[48] M. A. Barstow, D. D. Boyce, B. Y. Welsh, R. Lallement, J. K. Barstow, A. E. Forbes, and S. Preval, The Astrophysical Journal 723, 1762 (2010).

[49] R. F. Ferrero, C. M. Durán, J. L. Halbwachs, and A. M. C. Cubeiro, The Astronomical Journal 143, 28 (2011).

[50] J. X. Prochaska, B. Weiner, H. W. Chen, J. Mulchaey, and K. Cooksey, The Astrophysical Journal 740, 91 (2011).

[51] A. J. Fox, The Astrophysical Journal 730, 58 (2011).

[52] M. A. de Avillez and D. Breitschwerdt, Astronomy and Astrophysics 539, L1 (2012).

[53] G. Peach, H. E. Saraph, and M. J. Seaton, Journal of Physics B 21, 3669 (1988).

[54] Y. Ralchenko, A. E. Kramida, J. Reader, and N. A. Team, National Institute of Standards and Technology, Gaithersburg, MD (2012).

[55] A. Burgess, M. C. Chidichimo, and J. A. Tully, Astronomy and Astrophysics Supplement Series 131, 145 (1998).

[56] C. Allende Prieto, D. L. Lambert, and M. Asplund, The Astrophysical Journal 573, L137 (2002).

[57] C. Y. Chen, K. Wang, M. Huang, Y. S. Wang, and Y. M. Zou, Journal of Quantitative Spectroscopy and Radiative Transfer 111, 843 (2010).

[58] L. H. Andersen, G. Y. Pan, H. T. Schmidt, N. R. Badnell, and M. S. Pindzola, Physical Review A 45, 7868 (1992).

[59] L. Qu, Z. Wang, and B. Li, The European Physical Journal D 5, 173 (1999).

[60] J. M. Laming and U. Feldman, The Astrophysical Journal 591, 1257 (2003).

[61] M. Das, R. K. Chaudhuri, S. Chattopadhyay, U. S. Mahapatra, and P. K. Mukherjee, Journal of Physics B 44, 165701 (2011).

[62] M. Das, M. Das, R. K. Chaudhuri, and S. Chattopadhyay, Physical Review A 85, 042506 
(2012).

[63] R. K. Chaudhuri, S. Chattopadhyay, and U. Sinha Mahapatra, Physics of Plasmas 19, 2701 (2012).

[64] J. C. Stewart and K. D. Pyatt Jr, The Astrophysical Journal 144, 1203 (1966).

[65] D. Bielińska-Waż, J. Karwowski, B. Saha, and P. K. Mukherjee, Physical Review E 69, 016404 (2004).

[66] N. N. Dutta and S. Majumder, Physical Review A 85, 032512 (2012).

[67] A. I. Akhiezer, I. A. Akhiezer, R. A. Polovin, A. G. Sitenko, and K. N. Stepanov, Plasma Electrodynamics: Linear Response Theory, volume 1, Oxford: Pergamon, 1975.

[68] I. Lindgren and J. Morrison, Atomic many-body theory, Berlin Springer-Verlag, 1985.

[69] I. Lindgren and D. Mukherjee, Physics Reports 151, 93 (1987).

[70] M. A. Haque and D. Mukherjee, Journal of Chemical Physics 80, 5058 (1984).

[71] S. Pal, M. Rittby, R. J. Bartlett, D. Sinha, and D. Mukherjee, Chemical Physics Letters 137, $273(1987)$.

[72] S. Pal, M. Rittby, R. J. Bartlett, D. Sinha, and D. Mukherjee, Journal of Chemical Physics 88, 4357 (1988).

[73] E. Eliav, U. Kaldor, and Y. Ishikawa, Physical Review A 50, 1121 (1994).

[74] T. A. Isaev, A. N. Petrov, N. S. Mosyagin, A. V. Titov, E. Eliav, and U. Kaldor, Physical Review A 69, 30501 (2004).

[75] C. Sur, K. V. P. Latha, B. K. Sahoo, R. K. Chaudhuri, B. P. Das, and D. Mukherjee, Physical Review Letters 96, 193001 (2006).

[76] G. Dixit, B. K. Sahoo, R. K. Chaudhuri, and S. Majumder, Physical Review A 76, 042505 (2007).

[77] G. Dixit, H. S. Nataraj, B. K. Sahoo, R. K. Chaudhuri, and S. Majumder, Physical Review A 77, 012718 (2008).

[78] G. Dixit, H. S. Nataraj, B. K. Sahoo, R. K. Chaudhuri, and S. Majumder, Journal of Physics B 41, 025001 (2008).

[79] R. Pal, M. S. Safronova, W. R. Johnson, A. Derevianko, and S. G. Porsev, Physical Review A 75, 042515 (2007).

[80] B. K. Mani and D. Angom, Physical Review A 81, 042514 (2010).

[81] A. Szabo and N. S. Ostlund, Modern quantum chemistry: introduction to advanced electronic 
structure theory, Dover Pubns, 1996.

[82] I. P. Grant, Journal of Physics B 7, 1458 (1974).

[83] W. R. Johnson, D. R. Plante, and J. Sapirstein, Advances In Atomic, Molecular, and Optical Physics, 255 (1995).

[84] R. K. Chaudhuri, P. K. Panda, H. Merlitz, B. P. Das, U. S. Mahapatra, and D. Mukherjee, Journal of Physics B 33, 5129 (2000).

[85] F. A. Parpia and A. K. Mohanty, Physical Review A 46, 3735 (1992).

[86] F. A. Parpia, C. F. Fischer, and I. P. Grant, Computer Physics Communications 175, 745 (2006).

[87] H. Elabidi, S. Sahal-Bréchot, M. S. Dimitrijević, and N. Ben Nessib, Monthly Notices of the Royal Astronomical Society 417, 2624 (2011).

[88] C. Sur and R. K. Chaudhuri, Journal of Physics B 40, 4307 (2007).

[89] U. Feldman, Physica Scripta 24, 681 (1981).

[90] M. M. Dworetsky, C. M. Jomaron, and C. A. Smith, Astronomy and Astrophysics 333, 665 (1998).

[91] S. Sahoo and Y. K. Ho, Physics of Plasmas 13, 063301 (2006). 